\title{
Induced Endothelial Cells Enhance Osteogenesis and Vascularization of Mesenchymal Stem Cells
}

\author{
Jie Tao ${ }^{a}$ Yuan Sun ${ }^{b}$ Qiu-gen Wang ${ }^{a}$ Cheng-wen Liu ${ }^{c}$ \\ a Department of Orthopedics, Shanghai First People's Hospital, 'b Department of Orthopedics, \\ Shanghai Sixth People's Hospital, Shanghai Jiao-tong University, and 'Department of Orthopedics, \\ Branch of Shanghai First People's Hospital, Shanghai, China
}

\section{Key Words}

Vascularization $\cdot$ Mesenchymal stem cells $\cdot$ Endothelial cells · Osteogenesis

\begin{abstract}
Adequate vascularization remains one of the major challenges in bone tissue engineering. Since the microvascular endothelium is of benefit to osteogenesis and vascularization when in direct contact with bone marrow mesenchymal stem cells (BM-MSCs), we investigated whether endothelial cells induced from BM-MSCs have the same effect on BMMSCs in vitro and in vivo. BM-MSCs were isolated, characterized and induced into endothelial-like cells (induced endothelial cells, IECs) in endothelial cell growth medium 2. BM-MSCs and IECs were co-cultured with direct contact. In vitro, IECs were evaluated in terms of their characteristics of endothelial cells and their effects on the osteogenic potential of BM-MSCs by cell morphology, immunofluorescent staining, alkaline phosphatase activity and osteocalcin synthesis. In vivo, scaffolds consisting of $\beta$-tricalcium phosphate co-seeded with IECs and BM-MSCs were transplanted into mouse dorsal pockets, and a histological analysis was performed to determine the extent of new bone and blood vessel formation. Isolated BM-MSCs were positive for the mark-
\end{abstract}

Jie Tao and Yuan Sun contributed equally to this work. ers CD105 and CD29 and negative for hematopoietic markers CD34, CD45 and CD14. They were able to differentiate into adipocytes, osteocytes and chondrocytes in respective media. Immunofluorescent analysis with von Willebrand factor and CD31 staining showed that BM-MSCs could differentiate into endothelial cells. The alkaline phosphatase activity and the osteocalcin content of the co-culture group were obviously higher than those of any other group $(p<0.05)$. Histologically, newly formed bone and vessels were more evident in the culture group $(p<0.05)$. Our findings suggest that IECS could efficiently stimulate the in vitro differentiation of osteoblast-like cells and promote osteogenesis in vivo by direct contact with BM-MSCs.

Copyright $\odot 2009$ S. Karger AG, Basel

\begin{tabular}{|c|c|}
\hline \multicolumn{2}{|c|}{ Abbreviations used in this paper } \\
\hline ALP & alkaline phosphatase \\
\hline BM-MSCs & bone marrow mesenchymal stem cells \\
\hline$\beta-\mathrm{TCP}$ & $\beta$-tricalcium phosphate \\
\hline DiI-Ac-LDL & $\begin{array}{l}1,1^{\prime} \text {-dioctadecyl-3,3,3',3'-tetramethylindocar- } \\
\text { bocyanine perchlorate }\end{array}$ \\
\hline DMEM-LG & low-glucose DMEM \\
\hline EGM2-MV & endothelial cell growth medium 2 \\
\hline $\mathrm{H} \& \mathrm{E}$ & hematoxylin and eosin \\
\hline IECs & induced endothelial cells \\
\hline $\mathrm{OCN}$ & osteocalcin \\
\hline vWF & von Willebrand factor \\
\hline
\end{tabular}

\section{KARGER}

Fax +41613061234 E-Mail karger@karger.ch www.karger.com
(C) 2009 S. Karger AG, Base

$1422-6405 / 09 / 1904-0185 \$ 26.00 / 0$

Accessible online at:

www.karger.com/cto
Dr. Jie Tao

Department of Orthopedics, Shanghai First People's Hospital

Shanghai Jiao-tong University

No. 85 Wujin Road, Shanghai 200080 (China)

Tel. +86 216436 9181, E-Mail david1979982@163.com 


\section{Introduction}

At present, in the research of tissue engineering, one challenge of particular importance is to enhance vascularization of tissue-engineered bone [Cancedda et al., 2007]. Different approaches have been developed to overcome this problem, such as the incorporation of pro-angiogenic growth factors into biomaterials [Perets et al., 2003], the generation of pro-angiogenic matrices [Geiger et al., 2005], or genetic modification of cells to secrete growth factors [Elbjeirami and West, 2006]. Nevertheless, these approaches might not reflect the complex process leading to the formation of functional vessels. In recent years, the culture of bone marrow mesenchymal stem cells (BM-MSCs) with endothelial cells has been proposed as an effective tool to address rapid vascularization of tissue-engineered grafts. For example, culture of BM-MSCs together with dermal microvascular endothelial cells improved osteogenesis and vascularization of BM-MSCs [Kaigler et al., 2005]. In addition, rat kidney vascular endothelial cells seeded with BM-MSCs could enhance blood perfusion and bone formation of the tissue-engineered constructs after transplantation [Sun et al., 2007].

However, the endothelial and osteoblastic cells used by the previous studies were derived from different sources or even species, which limited potential clinical applications with regard to rejection and inconvenience of the culture. To solve this problem, Scherberich et al. [2007] applied human stromal vascular fraction cells as a common source of osteoblastic and endothelial cells and achieved good results.

In this study, we used BM-MSCs as another common source of osteoblastic and endothelial cells for the engineering of bone grafts, and we induced BM-MSCs into endothelial cells and investigated whether endotheliallike cells (induced endothelial cells, IECs) have the same effects on BM-MSCs as dermal microvascular endothelial cells or kidney microvascular endothelial cells in vitro and in vivo.

\section{Materials and Methods}

\section{Culture of BM-MSCs}

Human bone marrow was collected from patients undergoing iliac bone graft procedures (with approval of the University of Shanghai Jiao-tong Institutional Review Board), and cells were isolated. Briefly, marrow was placed in low-glucose DMEM (DMEM-LG; Gibco, Carlsbad, Calif., USA) with $100 \mathrm{U} / \mathrm{ml}$ sodium heparin and centrifuged at $700 \mathrm{~g}$ for $10 \mathrm{~min}$; the cell pellet was then resuspended in fresh DMEM-LG. All preparations were pipetted repeatedly to break up cell aggregates. Subsequently, marrow cell suspensions were passed consecutively through 16.5 - and 20.5-gauge needles before culture. Cells were then cultured in T75 tissue culture flasks with $15 \mathrm{ml}$ of basic medium and incubated at $37^{\circ} \mathrm{C}$ in a humidified atmosphere containing $95 \%$ air and $5 \%$ $\mathrm{CO}_{2}$. The basic medium consisted of DMEM-LG containing $10 \%$ fetal bovine serum (Gibco) and antibiotics. The medium was changed to remove non-adherent cells $24 \mathrm{~h}$ later. When the cell cultures became almost confluent after 8 days, they were released by treatment with $0.25 \%$ trypsin containing $1 \mathrm{~mm}$ EDTA for $3 \mathrm{~min}$ at $37^{\circ} \mathrm{C}$. The cells were counted and subcultured in $75-\mathrm{cm}$ flask.

\section{Flow Cytometry}

Passage 3 BM-MSCs were trypsinized, washed with PBS and incubated with fluorescein isothiocyanate- or phycoerythrinconjugated monoclonal antibodies specific for Sca-1, CD105 (endoglin), CD29, CD90, CD45, CD34, CD14 or control isotype IgG on ice for $30 \mathrm{~min}$. All antibodies were purchased from BD Pharmingen, San Diego, Calif., USA. Analysis was performed by flow cytometry (Becton Dickinson, Franklin Lakes, N.J., USA) using Cell Quest software.

\section{BM-MSC Differentiation Assays}

Passage 3 BM-MSCs were incubated to differentiate into adipocytes, osteoblasts and chondrocytes in corresponding induction medium for 3 weeks. Adipogenic medium contained $10^{-6} \mathrm{M}$ dexamethasone, $10 \mu \mathrm{g} / \mathrm{ml}$ insulin and $100 \mu \mathrm{g} / \mathrm{ml} 3$-isobutyl-Lmethylxantine (Sigma-Aldrich, St. Louis, Mo., USA). Adipogenic differentiation was confirmed by the formation of neutral lipid vacuoles stainable with Oil Red O (Sigma-Aldrich). Osteogenic medium contained $10^{-8} \mathrm{M}$ dexamethasone, $50 \mu \mathrm{g} / \mathrm{ml}$ ascorbic acid and $10 \mathrm{~mm} \beta$-glycerophosphate (Sigma-Aldrich). Cultures were stained for alkaline phosphatase (ALP; alkaline phosphatase detection kit, Sigma-Aldrich). Chondrogenic differentiation medium contained $10 \mathrm{ng} / \mathrm{ml}$ transforming growth factor- $\beta_{1}, 6.25$ $\mathrm{mg} / \mathrm{l}$ insulin and $1 \%$ antibiotic/antimycotic. Cells were stained with $1 \%$ toluidine blue (Sigma-Aldrich).

\section{Endothelial Cell Differentiation}

Confluent cells were trypsinized, washed with PBS and cultivated in endothelial cell growth medium 2 (EGM2-MV; Clonetics, San Diego, Calif., USA) with 10\% fetal bovine serum (Gibco) for 14 days. The medium was changed every 2 days.

\section{Immunofluorescent Staining}

For von Willebrand factor (vWF) and CD31 staining, cells were fixed with methanol at $-20^{\circ} \mathrm{C}$ for $10 \mathrm{~min}$ and rinsed with PBS. Samples were incubated with an antibody against vWF and CD31 (Merck, Darmstadt, Germany) for 30 min, rinsed with PBS and incubated with a labeled secondary antibody.

In vitro Groups of the Experiment

BM-MSCs and IECs were cultured either with direct contact or separately. In the direct contact or BM-MSCs/IECs setup, BMMSCs $\left(1 \times 10^{4} \mathrm{cells} / \mathrm{ml}\right)$ were co-cultured with IECs on the inserts of 6-well plates at a 1:1 ratio. The medium used was the osteogenic medium which consisted of the basic medium supplemented with $50 \mu \mathrm{g} / \mathrm{ml}$-ascorbic acid, $10 \mathrm{mM} \beta$-glycerophosphate, 
$10^{-8} \mathrm{M}$ dexamethasone (Sigma). In the BM-MSCs setup, BMMSCs $\left(1 \times 10^{4}\right.$ cells $\left./ \mathrm{ml}\right)$ were separately cultured on the inserts of 6 -well plates with the osteogenic medium. In the IECs setup, IECs $\left(1 \times 10^{4}\right.$ cells $\left./ \mathrm{ml}\right)$ were cultured on the inserts of 6 -well plates with EGM2-MV. All studies used cells below their sixth passage in culture.

\section{Analysis of Osteogenesis in vitro}

ALP assays were performed to measure early osteoblastic differentiation of BM-MSCs. Cells were lysed by using passive lysis buffer (Promega, Madison, Wisc., USA), and lysates were sonicated for 4-5 s and transferred to ice. Samples were centrifuged for $10 \mathrm{~min}$, and $100 \mu \mathrm{l}$ of the supernatant was collected. The remaining volume of supernatant and lysate was used for DNA analysis (Hoechst Dye Assay; Sigma-Aldrich). ALP activity in samples was quantified by means of a colorimetric assay. ALP activity values were normalized to the amount of BM-MSCs DNA, not total cellular DNA in a sample, to obtain units of ALP/mg DNA. Staining was also performed for ALP with cells fixed in $70 \% \mathrm{EtOH}$ as described previously. Four independent culture experiments were performed.

\section{Cell Number Normalization in Co-Cultures}

Total DNA was collected from samples and quantified by the Hoechst Dye Assay. In culture experiments, the number of BMMSCs was determined to allow normalization of ALP and osteocalcin (OCN) levels to BM-MSC cell number, instead of total cell number, to account for the 2 cell types in culture assays. To determine the ratios of endothelial cells to BM-MSCs in these culture DNA samples, the following measure was used [Kaigler et al., 2005]. First, staining with acetylated low-density lipoprotein, labeled with 1,1'-dioctadecyl-3,3,3',3'-tetramethylindocarbocyanine perchlorate (DiI-Ac-LDL; Biomedical Technologies, Stoughton, Mass., USA), was performed in Lab-Tek chamber slides (Nalge Nunc International, Rochester, N.Y., USA) on parallel cultures to qualitatively determine the contribution of endothelial cells to the total cell number in co-cultures. DiI-Ac-LDL was diluted to $10 \mu \mathrm{g} / \mathrm{ml}$ in EGM2-MV, added to cultures and allowed to incubate for $4 \mathrm{~h}$ at $37^{\circ} \mathrm{C}$. The medium was removed and cultures were rinsed 3 times with PBS. Cells were then fixed in 3\% formaldehyde in PBS for $20 \mathrm{~min}$ at room temperature. After fixation, cells were rinsed in deionized water and excess liquid was drained. Wells were removed from Lab-Tek chamber slides and covered with coverslips, with $90 \%$ glycerol and $10 \%$ PBS, and the numbers of positive and total cells were counted.

\section{OCN ELISA}

A Mid-Tact Human OCN ELISA Kit (Biomedical Technologies) was used for the detection of human OCN secreted by BMMSCs. BM-MSCs were grown alone or in culture with IECs for 14 days. The medium was changed 1 day after cell seeding and every 2 days thereafter. Seven and 14 days after cell seeding, 12-hour culture medium was collected from both culture conditions and measured for the presence of OCN. Three independent culture experiments were performed.

\section{Cell Seeding and Scaffold Transplantation}

The porous $\beta$-tricalcium phosphate $(\beta$-TCP) scaffolds were provided by BIO-LU Medical (Shanghai, China). The average pore size of this scaffold is $200-400 \mathrm{~mm}$ in diameter. Almost all pores are interconnected via a 100 - to $200-\mathrm{mm}$ path. The average porosity is $75 \pm 3 \%$. The porous $\beta$-TCP was cut into blocks of $5 \times 5 \times 5 \mathrm{~mm}$. Before these blocks were combined with cells, they were sterilized in an autoclave at $180^{\circ} \mathrm{C}$ for $4 \mathrm{~h}$.

Severe combined immunodeficient mice (7-9 weeks old) were first anesthetized, and a $2 \times 2 \mathrm{~cm}$ area was shaved clean on their back. A small (about 1.5- $\mathrm{cm}$ ) longitudinal skin incision was then made in the center of the shaved area. The tips of the dissecting scissors were slipped under the skin (between the skin and muscle layers) to bluntly dissect subcutaneous fascial tissue and create pockets. Samples of $50 \mu \mathrm{l}$ of cell suspensions, containing $5 \times 10^{5}$ cells of the appropriate cell type for each condition, were added to each scaffold with a Pipetman. Porous $\beta$-TCP containing BMMSCs/IECs or BM-MSCs alone was cultured for 1 week before being implanted into the right or left dorsal pocket of each animal. Implants were placed, and the incisions were closed with interrupted sutures. At 4 and 8 week, animals were sacrificed and implants were harvested for evaluation of bone and blood vessel formation.

Tests of Bone and Blood Vessel Formation in vivo:

Tissue Preparation and Histological Staining

Implants were retrieved at various time points and fixed overnight (about $16 \mathrm{~h}$ ) in aqueous buffered zinc formalin. Implants were then transferred to $70 \% \mathrm{EtOH}$ until processed and embedded in paraffin. Paraffin-embedded matrices were cut into serial sections (5 $\mu \mathrm{m}$ thick) and placed on glass slides for histological testing. Tissue sections were stained with hematoxylin and aqueous eosin Y solution (H\&E; Sigma-Aldrich) to visualize the overall tissue morphology.

\section{Quantification of Blood Vessels and Bone}

Tissue sections were visualized and photographed with the E-800 light microscope (Nikon, Melville, N.Y., USA). Five samples from each condition were analyzed manually and digitally. The total amounts of blood vessels present in the implants were analyzed. Blood vessels were identified in $\mathrm{H} \& \mathrm{E}$-stained tissues (at $\times 100$ magnification), by defined lumens and the presence of red blood cells within their boundaries. Bone histomorphometric analysis of sections stained with $\mathrm{H} \& \mathrm{E}$ was performed to quantify bone formation. By means of an E-800 light microscope, histological sections from each implant at each time point were scanned and imported into Adobe Photoshop (Adobe Systems). The bone tissue area for each section was determined by dividing the total number of bone pixels by the total number of implant pixels based on color by using ImagePro Plus software (Media Cybernetics, San Diego, Calif., USA). This value was plotted as bone area/tissue area. Identification of bone was based on morphology of eosinstained tissue and the identification of cell lining (osteoblasts) and within (osteocytes) this tissue.

\section{Statistical Analysis}

Average values were expressed as the arithmetic means \pm SD. The analysis of data was performed by the paired t test. Differences were considered statistically significant when the $\mathrm{p}$ value was $<0.05$. 


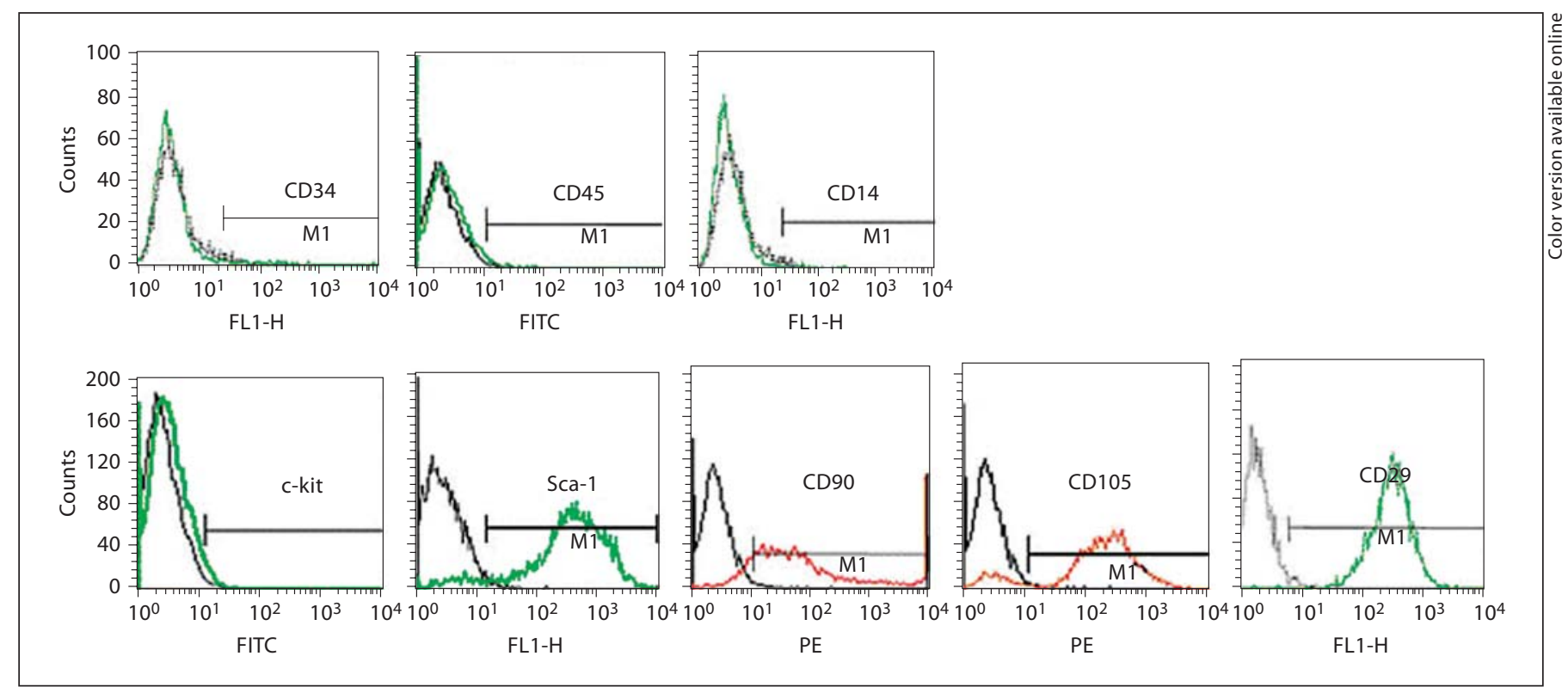

Fig. 1. FACS analysis of typical hematopoietic markers which are not expressed by MSCs (upper line) and typical CD markers present on BM-MSCs (lower line).

\section{Results}

\section{In vitro Studies}

Cell Culture of BM-MSCs

BM-MSCs were isolated according to standard technique for the isolation of mononuclear cells from bone marrow. Phase contrast microscopy from cells demonstrated a fibroblast-like, spindle-shaped morphology. Cells were tested with flow cytometry for the presence or absence of characteristic hematopoietic and endothelial markers. BM-MSCs typically expressed the antigens CD105 and CD29. Furthermore, cells expressed Sca-1 and CD90. They were negative for typical lymphocytic markers like CD45 and CD14, as well as for the early hematopoietic marker CD34 (fig. 1). The ability of the BM-MSCs to differentiate into osteocytes, adipocytes and chondrocytes was confirmed by different staining (fig. 2).

\section{Histological Evaluation of IECs}

The BM-MSCs maintained in differentiation culture for about 2 weeks had a 'rounded' shape before digestion. Cells subsequently attached to the wall of the culture flask after about $6 \mathrm{~h}$ displayed a polygonal shape. Upon reaching an $80-90 \%$ confluence, cells had a typical 'cobblestone-like' appearance under light microscopy (fig. 3c).
Immunohistochemistry data suggest that over $90 \%$ of differentiating cells from MSCs stained positively for vWF and CD31 (fig. 3d-f). These results demonstrate that the cells derived from human BM-MSCs were endothelial cells.

\section{In vitro Effects of IECs on BM-MSC Osteogenic}

Differentiation

To determine the effects of IECs on BM-MSC osteogenic differentiation, BM-MSCs were co-cultured with IECs. ALP expression was used as an early measure of osteogenic differentiation. Quantitative analysis of ALP activity confirmed the qualitative observations as there was a statistically significant $(\mathrm{p}<0.05)$ increase (as much as 2 fold) in ALP activity in BM-MSC/IEC cultures compared with BM-MSCs alone (fig. 4a). IECs cultured alone were assayed for ALP activity. IECs alone showed very minimal baseline levels of ALP.

As an additional measure of BM-MSC osteogenic differentiation in co-culture conditions, a later-stage osteogenic marker, OCN was quantified. At the second week, the osteocalcin content of the culture group was obviously higher than that of any other group ( $p<0.05$; fig. $4 \mathrm{~b}$ ). OCN from IECs was also measured, but no detectable levels were present in endothelial cell culture medium (data not shown). 

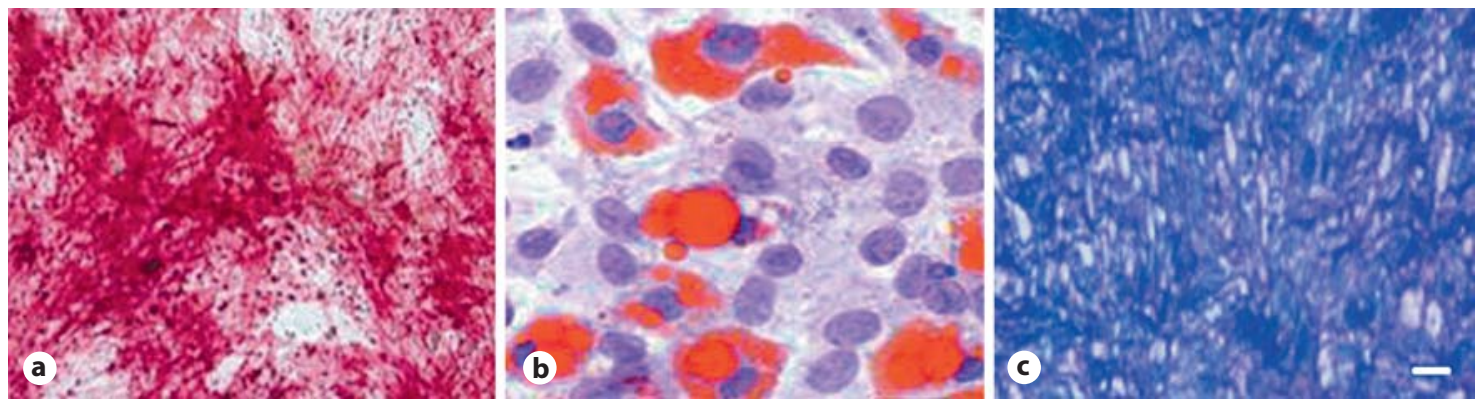

Fig. 2. Differentiation of BM-MSCs. Cultured in appropriate differentiation media, BM-MSCs differentiated into osteoblasts (a), which expressed ALP, indicated in red, adipocytes (b), which are indicated by accumulation of lipid vesicles indicated by Oil Red O stain in the cells, and chondrocytes (c), indicated in blue after toluidine blue stain. Scale bar $=50 \mu \mathrm{m}$.
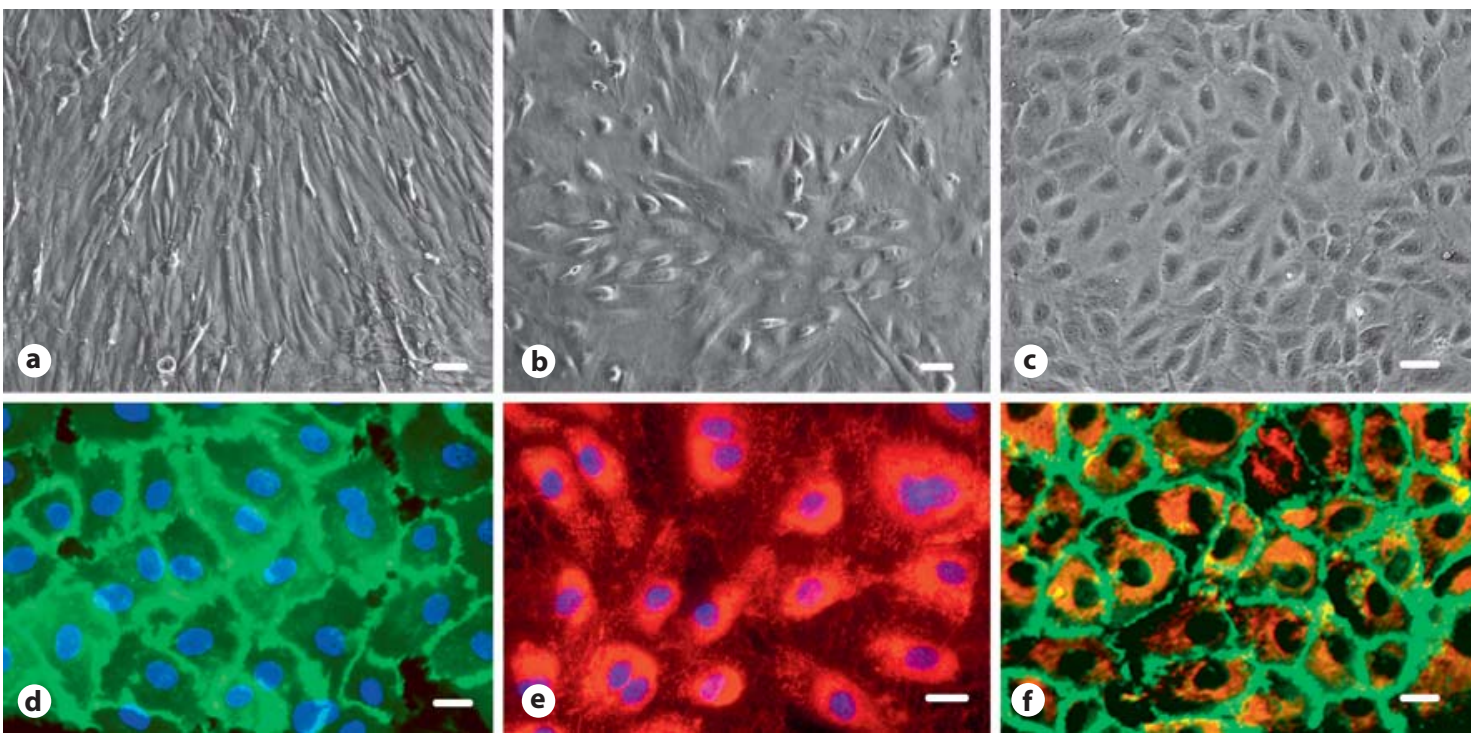

Fig. 3. a BM-MSCs under phase contrast microscopy. b BM-MSCs after 7 days differentiation into endothelial cells. c After 14 days, differentiating endothelial cells derived from mesenchymal stem cells formed a confluent monolayer with a typical 'cobblestone-like' appearance. $\mathbf{d}$ IECs stained positively for vWF. e IECs stained positively for CD31. f IECs stained positively for vWF and CD31. Scale bars = 5 $\mu \mathrm{m}(\mathbf{a}), 10 \mu \mathrm{m}(\mathbf{b}, \mathbf{c}), 20 \mu \mathrm{m}(\mathbf{d}-\mathbf{f})$.

\section{In vivo Effect of IECs on BM-MSC Osteogenic}

\section{Potential}

Blood Vessel Analysis at Week 4 and 8

To determine whether IECs enhance BM-MSC osteogenic differentiation in vivo, BM-MSCs were co-transplanted with IECs. Blood vessels were analyzed to determine whether transplanted IECs enhanced neovascularization within the scaffolds. Four and 8 weeks after implantation, samples were retrieved and analyzed for neovascularization within the scaffold by means of functional blood vessel count. At 4 weeks after implantation, there was a not significantly higher number of vessels in scaffolds containing human BM-MSCs and IECs than in scaffolds containing BM-MSCs alone, while at 8 weeks after implantation, BM-MSCs/IECs showed statistically significant increases in the angiogenic response compared with the BM-MSCs alone (fig. 5e).

Bone Test at Week 4 and 8

Bone formation within the scaffolds was also measured at 4 and 8 weeks after transplantation. Histological examination revealed localized regions of bony islands in 


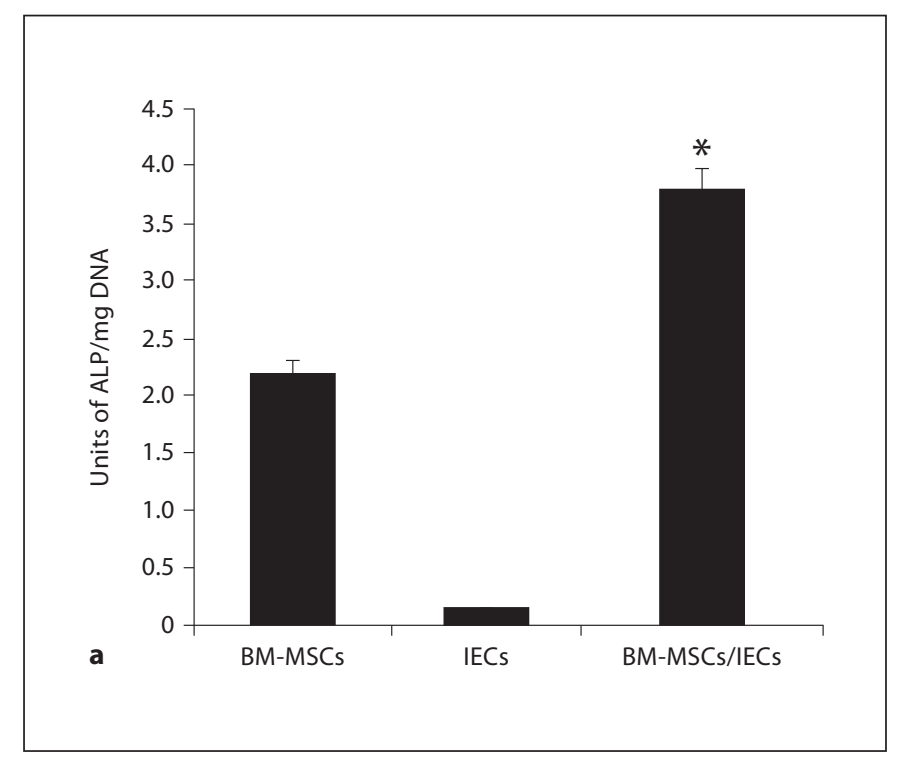

Fig. 4. a Quantitative analysis of ALP expression from BM-MSCs cultured for 2 weeks, alone or at 1:1 ratios with IECs. ALP expression was also measured for endothelial cells grown alone in culture. ${ }^{*} \mathrm{p}<0.05$, compared with the BM-MSC condition. b OCN production from BM-MSCs was measured from BM-MSC-conditioned medium after 7 and 14 days. BM-MSCs were cultured

both conditions. At 4 weeks, comparison of bone tissue histomorphometry from these scaffolds revealed a 7\% increase in bone formation in scaffolds containing human BM-MSCs and IECs relative to scaffolds containing BMMSCs alone. However, this increase in bone formation was not statistically significant $(\mathrm{p}<0.1)$. At 8 weeks after transplantation, in the evaluation of bone tissue formation, gross qualitative histological analysis of whole sections suggested that scaffolds transplanted with BMMSCs and IECs formed more bone tissue than scaffolds transplanted with BM-MSCs alone (fig. 5b, c). Bone tissue had a wavy, trabecular appearance with little to no presence of hematopoietic tissue formation. Quantitative bone histomorphometry revealed a statistically significant $14 \%$ increase in engineered bone formation in implants containing both cell types compared with implants containing only BM-MSCs (fig. 5f).

\section{Discussion}

Bone defect repair is one of the major targets of tissue engineering research. Pre-seeding a biomaterial with tissue-specific cells prior to implant is one method which is

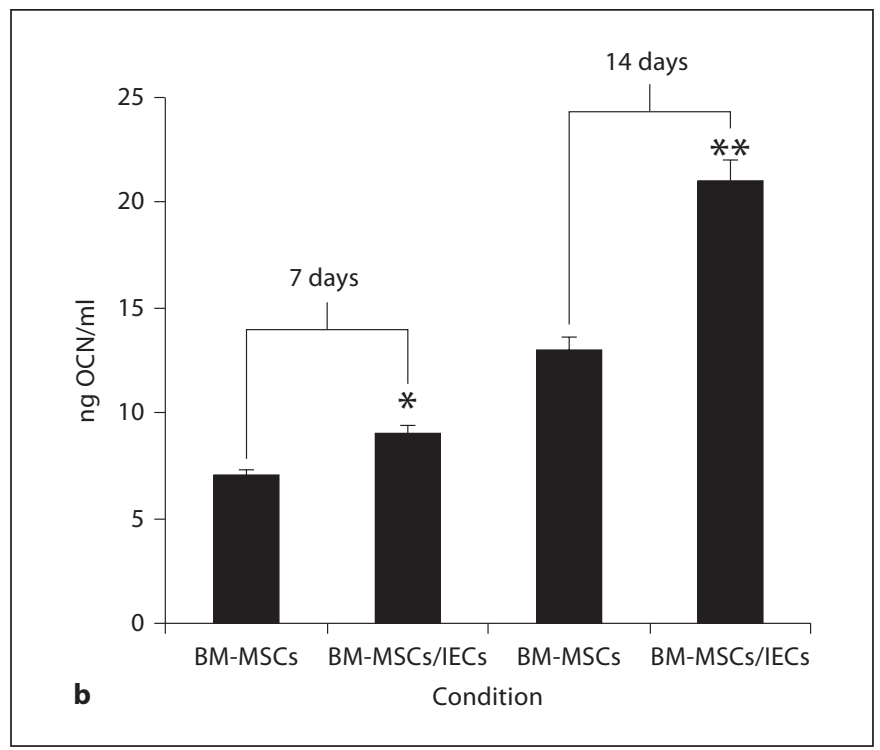

alone or co-cultured with IECs. OCN from IECs alone was also measured, but undetectable levels were present in endothelial cell culture medium. Values represent means $\pm \mathrm{SD}(\mathrm{n}=3) .{ }^{*} \mathrm{p}<0.05$, compared with the BM-MSC 7-day condition; ${ }^{* *} \mathrm{p}<0.05$, compared with the BM-MSC 14-day time point for OCN ELISA.

being examined to speed up tissue regeneration [Yancopoulos et al., 2000]. However, one of the major problems after the implantation of large biomaterials is the slow vascularization of the material [Akita et al., 2004]. One approach is to pre-seed the material with not only tissuespecific cells but also with endothelial cells. For example, Levenberg and colleagues [2005] have shown that culture of myoblasts together with human umbilical vein endothelial cells improved blood perfusion and survival of the muscle tissue constructs after transplantation. Tremblay et al. [2005] reported that capillary-like structures formed in vitro by culture of keratinocytes and human umbilical vein endothelial cells were able to create a connection with the host vessels upon implantation, thus accelerating blood supply of hypoxic zones within the skin graft. Similarly, Kaigler et al. [2005] also demonstrated that human dermal microvascular endothelial cells cultured with osteoblastic lineage cells could form an extensive microcapillary-like network in vitro and enhance bone formation. However, in these studies, the endothelial cells were derived from different sources or even species, which leads to rejection in vivo and limits the potential clinical applications. At the same time, the clinical use of mature endothelial cells derived from autologous vascu- 
Fig. 5. a-d In vivo experiments; $H \& E$ staining of tissue taken from the implanted area, and quantitative measurements of new blood vessel and new bone from $\mathrm{H} \& \mathrm{E}$ staining slides. Scale bars $=100 \mu \mathrm{m}$. a $\beta$-TCP/BM-MSC/IEC composite 4 weeks after implantation. $\mathrm{P}=1$ pore; $\mathrm{T}=$ ghost of $\beta$-TCP produced by decalcification; $\mathrm{B}=$ bone formation inside the pores. $\mathbf{b} \beta-\mathrm{TCP} /$ BM-MSC composite 8 weeks after implantation. $\mathrm{T}=$ Ghost of $\beta$-TCP produced by decalcification; $\mathrm{B}=$ mature osteocytes in pores; $\mathrm{V}=$ blood vessels through interconnecting paths; $\mathrm{R}=$ red cells in blood vessels; $\mathrm{O}=$ osteoblasts. c $\beta$-TCP/BM-MSC/ IEC composite 8 weeks after implantation. $\mathrm{B}=$ Osteocytes; $\mathrm{V}=$ blood vessels; $\mathrm{N}=$ multinuclear cells; $\mathrm{O}=$ osteoblasts. d Higher magnification view of the rectangular area in c. $\mathrm{O}=$ Osteoblasts; $\mathrm{B}=$ osteocyte; $\mathrm{V}=$ blood vessel. e Analysis of new blood vessels formed 8 weeks after implantation of scaffolds containing BM-MSCs alone or BM-MSCs and IECs. f Analysis of bone formed 8 weeks after implantation of scaffolds containing BMMSCs alone or BM-MSCs and endothelial cells. In H\&E-stained sections, bone and blood vessels were measured as a percentage of total tissue area within the scaffold. Values represent means $\pm \mathrm{SD}(\mathrm{n}=3)$. ${ }^{*} \mathrm{p}<0.05$, compared with the BM-MSC condition.
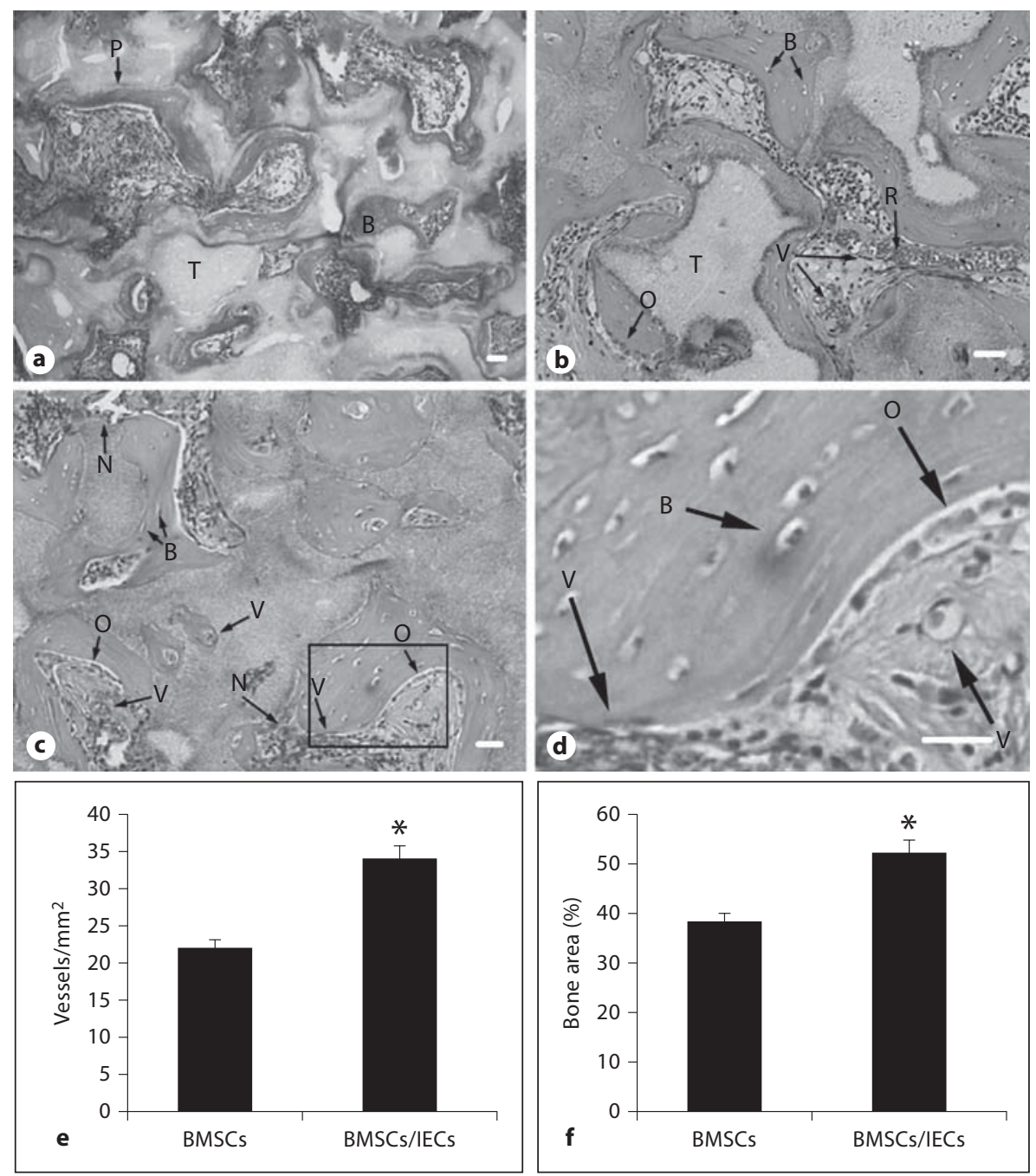

lar tissue presents some important limitations: (1) the isolation relies on an invasive procedure; (2) mature endothelial cells show relatively low proliferative potential; and (3) it is difficult to obtain a sufficient number of cells from a small biopsy of autologous tissue. In addition, in the context of skeletal or skin tissue engineering, tissue vascularization by implanted endothelial cells is strongly dependent on the co-seeding of fibroblasts. In our study, we used BM-MSCs as cell source to acquired endothelial cells and confirmed that in vitro and in vivo IECs enhanced osteogenesis and vascularization without the need of co-implanting supporting perivascular cells such as fibroblast. For clinical applications, autologous IECs from BM-MSCs are more attractive and suitable with regard to the easy availability of a large number of functional cells and the minimally invasive harvest method.
BM-MSCs are known to include progenitor cells with extensive plasticity and a capacity to differentiate into various musculoskeletal lineages as well as into lipocytes, osteocytes and chondrocytes. The high proliferation rate of these cells allows their expansion up to an adequate cell number for tissue engineering applications [Fuchs et al., 2006]. Our study shows that in our endothelial differentiation system, human bone marrow-derived (CD105+, CD29+, CD34-) MSC are capable of differentiating into endothelial cells in vitro and acquire major characteristics of mature endothelial-like expression of vWF and CD31. This will make BM-MSCs attractive candidates for the development of autologous tissue graft.

IECs enhance the osteogenic potential of BM-MSCs when grown in culture in vitro compared with BM-MSCs alone (fig. 2a). In the direct co-culture, there was a con- 
sistent enhancement in osteogenic differentiation of BMMSCs as measured by ALP activity and OCN production from BM-MSCs. This finding is identical with the previous report that osteoblastic progenitor cells derived from bone marrow increased ALP activity when in direct contact with human dermal microvascular endothelial cells [Kaigler et al., 2005].

As we know, transplanted endothelial cells have the ability to form blood vessels and could potentially increase neovascularization to regenerating tissues [Unger et al., 2007]. In the present study, 8 weeks after implantation, the BM-MSC/IEC condition showed statistically significant increases in the total number of blood vessels compared with the BM-MSCs alone. One reason for this finding could be a maximal angiogenic response from the endothelial cell production of vascular endothelial growth factor [Li et al., 2000; Villars et al., 2002]. Another important reason for this may be a direct effect of IECs in blood vessel formation. Kaigler et al. [2005] reported that human dermal microvascular endothelial cells directly form blood vessels in vivo when they are co-implanted with BM-MSCs [Kaigler et al., 2005]. Recent work from Scherberich et al. [2007] also suggested that the transplanted cells of the endothelial lineage present in the grafts were capable of generating blood vessels in vivo. These observations indicate that the in vivo formation of microvessels is in part dependent on implanted endothelial cells. In our experiment, no matter which reason takes effect, the addition of IECs enhances vascularization of the scaffold. In the future, a further study will be needed to investigate the mechanism of blood vessel formation in the culture condition. The other finding that total blood vessel formation was more obvious was reported in another study [Kaigler et al., 2005]. This may have been caused by the characteristics of induced endothelial cells used in our studies which have a high proliferation rate since they come from adult stem cells, or by the different property of scaffold affecting angiogenic response elicited by transplanted endothelial cells.

Not only did IECs enhance vascularization in vivo but they can increase bone formation in vivo. This result suggests that the transplanted endothelial cells had stimulatory effects on the ability of BM-MSCs to form bone. One likely explanation for this finding is that endothelial cells directly enhance osteogenic differentiation of BM-MSCs, as has been demonstrated in vitro. The proximity of these cell types to one another when seeded onto $\beta$-TCP scaffolds lends itself to cell-cell interactions and communication between endothelial cells and BM-MSCs which could contribute to the potential of the bone-forming ability of BM-MSCs [Bouletreau et al., 2002; Furumatsu et al., 2003]. Another reason is indirect effects on bone formation through a heightened angiogenic response.

In conclusion, our results demonstrate that BM-MSCs could differentiate into endothelial cells and IECs and not only enhance vascularization but also increase bone formation in engineered bone tissue by direct contact with BM-MSC in vivo.

\section{Acknowledgments}

This work was supported by a grant from Shanghai Baoshan Foundation for Natural Sciences.

\section{References}

Akita, S., N. Tamai, A. Myoui, M. Nishikawa, T. Kaito, K. Takaoka, H. Yoshikawa (2004) Capillary vessel network integration by inserting a vascular pedicle enhances bone formation in tissue-engineered bone using interconnected porous hydroxyapatite ceramics. Tissue Eng 10: 789-795.

Bouletreau, P.J., S.M. Warren, J.A. Spector, Z.M. Peled, R.P. Gerrets, J.A. Greenwald, M.T. Longaker (2002) Hypoxia and VEGF up-regulate BMP-2 mRNA and protein expression in microvascular endothelial cells: implications for fracture healing. Plast Reconstr Surg 109: 2384-2397.
Cancedda, R., P. Giannoni, M. Mastrogiacomo (2007) A tissue engineering approach to bone repair in large animal models and in clinical practice. Biomaterials 28: $4240-$ 4250.

Elbjeirami, W.M., J.L. West (2006) Angiogenesis-like activity of endothelial cells co-cultured with VEGF-producing smooth muscle cells. Tissue Eng 12: 381-390.

Fuchs, S., M.I. Hermanns, C.J. Kirkpatrick (2006) Retention of a differentiated endothelial phenotype by outgrowth endothelial cells isolated from human peripheral blood and expanded in long-term cultures. Cell Tissue Res 326: 79-92.

\footnotetext{
Furumatsu, T., Z.N. Shen, A. Kawai, K. Nishida, H. Manabe, T. Oohashi, H. Inoue, Y. Ninomiya (2003) Vascular endothelial growth factor principally acts as the main angiogenic factor in the early stage of human osteoblastogenesis. J Biochem (Tokyo) 133: 633-639.

Geiger, F., H. Bertram, I. Berger (2005) Vascular endothelial growth factor gene-activated matrix (VEGF165-GAM) enhances osteogenesis and angiogenesis in large segmental bone defects. J Bone Miner Res 20: 20282035 .
} 
Kaigler, D., P.H. Krebsbach, E.R. West, K. Horger, Y.C. Huang, D.J. Mooney (2005) Endothelial cell modulation of bone marrow stromal cell osteogenic potential. FASEB J 19: 665692.

Levenberg, S., J. Rouwkema, M. Macdonald, E.S. Garfein, D.S. Kohane, D.C. Darland, R. Marini, C.A. Blitterswijk, R.C. Mulligan, P.A. D’Amore, R. Langer (2005) Engineering vascularized skeletal muscle tissue. Nat Biotechnol 23: 879-884.

Li, W.M., W.Q. Huang, Y.H. Huang, D.Z. Jiang, Q.R. Wang (2000) Positive and negative hematopoietic cytokines produced by bone marrow endothelial cells. Cytokine 12: 10171023.
Perets, A., Y. Baruch, F. Weisbuch, G. Shoshany, G. Neufeld, S. Cohen (2003) Enhancing the vascularization of three-dimensional porous alginate scaffolds by incorporating controlled release basic fibroblast growth factor microspheres. J Biomed Mater Res A 65: 489-497.

Scherberich, A., R. Galli, C. Jaquiery, J. Farhadi, I. Martin (2007) Three-dimensional perfusion culture of human adipose tissue-derived endothelial and osteoblastic progenitors generates osteogenic constructs with intrinsic vascularization capacity. Stem Cells 25: 1823-1829.

Sun, H.C., Z. Qu, Y. Guo, G.X. Zang, B. Yang (2007) In vitro and in vivo effects of rat kidney vascular endothelial cells on osteogenesis of rat bone marrow mesenchymal stem cells growing on polylactide-glycoli acid (PLGA) scaffolds. Biomed Eng 6: 41.

Tremblay, P.L., V. Hudon, F. Berthod, L. Germain, F.A. Auger (2005) Inosculation of tissue-engineered capillaries with the host's vasculature in a reconstructed skin trans planted on mice. Am J Transplant 5: 1002 1010.
Unger, R.E., A. Sartoris, K. Peters, A. Motta, C. Migliaresi, M. Kunkel, U. Bulnheim, J. Rychly, C.J. Kirkpatrick (2007) Tissue-like selfassembly in cocultures of endothelial cells and osteoblasts and the formation of microcapillary-like structures on three-dimensional porous biomaterials. Biomaterials 28: 3965-3976.

Villars, F., B. Guillotin, T. Amédée, S. Dutoya, L. Bordenave, R. Bareille, J. Amédée (2002) Effect of HUVEC on human osteoprogenitor cell differentiation needs heterotypic gap junction communication. Am J Physiol 282: 775-785.

Yancopoulos, G.D., S. Davis, N.W. Gale (2000) Vascular specific growth factors and blood vessel formation. Nature 407: 242-248. 